\title{
Optimization of Electrical Control System for Mechatronics Integrated Assembly Equipment
}

\author{
Yuelin Xu \\ Suzhou Institute of Industrial Technology, Suzhou City, Jiangsu 215000, China \\ xuyuelin@126.com
}

Keywords: mechatronics; assembly equipment; electrical control.

\begin{abstract}
China's machinery manufacturing industry is the rapid development of science and technology, especially with the continuous improvement, has realized the automation of assembly, through the integration of the integrated assembly device to control, optimization of the electrical control system, greatly reducing the labor output, but also can improve the production efficiency. In view of the needs of society, the current Mechatronics integrated assembly device is still optimizing the electric control system, so as to further meet the needs of the society, so as to improve the automatic water products of our Mechatronics integrated device. Based on this, this article from the reliability and safety of the electromechanical integrated assembly device with the first analysis of the optimization idea of Mechatronics integrated assembly device of the electric control system, and then study the main advantages of electromechanical integrated assembly device of the electric control system optimization strategy and optimization of the improved device, in order to provide some suggestions to the related study.
\end{abstract}

\section{Reliability and safety of Mechatronics integrated assembly device}

Mechatronics integrated assembly device is usually composed of IPC measurement system, sensor, mechanical body and pneumatic system. With the development of new era, CNC system is added, which can manage sensor and manage signal in real time. After adding CNC system, the operation of the whole mechanism can achieve multiple automation. Simple actions such as grabbing, moving and placing can perform autonomously, reducing manual output, and improving production efficiency and cost quality.

In industrial production, the reliability of product quality is a problem that people attach much importance to. Especially for some military equipment or special industry products, there are relatively strict technical indicators. The reliability of products can be generally divided into inherent reliability and application reliability. Application reliability mainly describes the reliability of its use in the environment of product planning, and its reliability is used to describe the reliability level of design and manufacture. In mechatronics integrated assembly of electrical control device, the reliability analysis is to improve the reliability of the whole system, a detailed analysis of the fault of each unit, especially around may affect the quality of the product failure, to one by one to explore and analyze the causes, improvement in the design, in order to improve the reliability of products. Many new parts are assembled by multiple tasks, and any one of the details may cause the quality of the whole product to be unqualified.

For the integrated assembly device of mechatronics, the security problem mainly refers to the safety of the operator and the safety of the assembled workpiece. Before starting the whole job processing, we should analyze the safety of the electrical system, evaluate the reliability of the control system, and take corresponding protective measures at the same time. Only by improving the reliability of the whole system, can the safety be guaranteed. For some cases of power failure, some precautions should be taken, for example, the vacuum suction cup should be controlled with two signal detection ends. Through these measures to minimize the impact of accidents on the entire electrical control system and improve the safety of production. 


\section{Optimization idea of electrical control system for integrated mechanical and electrical integrated assembly equipment}

In view of the actual application of the mechatronics integrated assembly device and the needs of the society, the optimization of its electrical control system can start from the following aspects.

(1) the optimization is considered from the actual assembly. Assembling device after the design is completed, but also verify the functionality, determine whether the basic functions to meet the design requirements, especially the characteristics to consider the assembly of products, according to the specific problems during experiment was improved, the only way we can ensure that the electric control system can effectively meet the demand of the production, implementation the optimization of the whole system.

(2) verification of security and reliability. After completing the system design, we must verify the reliability and safety and improve the stability of the device without cutting down its original functions. During reliability and safety verification, we can use derating design and redundancy design to realize, especially for some important parts, we must increase the process of safety detection. Aiming at the whole electrical control system, we can simplify the control structure and make the hardware more compact, which is more convenient to control, and also helps to improve the security of the system.

(3) the optimization of control software. For the software of the electrical control system, the degree of automation must be high, and the corresponding alarm information can be provided in the case of failure. In this case, the abnormal cause can be judged in time, and the fault is located, which is beneficial to the quick finding of the solution. For some tedious CNC systems, it can be simplified properly, reducing the difficulty of software development, increasing the simplicity of control and reducing the workload of optimization, which will also improve the flexibility of technology.

(4) the optimization of line layout. For the integrated assembly device of mechatronics, the internal electrical control system is very complex and the connection is more. In view of this situation, must carry on the optimization layout of the corresponding line, avoid miscellaneous lines caused by the use of risk, once the fault maintenance is very inconvenient. Under the current technology environment, sensors and PLC systems can be used to realize the optimization of line layout, minimize the internal space occupied by the line and reduce the difficulties of subsequent maintenance.

\section{Optimization strategy for electrical control system of integrated mechanical and electrical integrated assembly device}

\subsection{Redundant design}

Redundancy design is very common in electrical system optimization and improvement, for example, the opening and closing of manual machine tools. Because of the complexity of its system and work, it is difficult to achieve vacuum when the action is completed. In order to avoid the vacuum suction out the wrong action, when the opening or closing action should be to maintain the reliability of the circuit can be used on a dual output signal module means to control, so the design of parallel redundancy hybrid can improve the safety of use.

In the electrical control system of the other used cylinder device, can also be modeled by this model, so that the two modules work at the same time, and each signal output control relay in parallel, as long as there is a small relay failure, can obtain the analog signal is needed, this makes it possible to complete the appropriate action in accordance with the the expected design procedure, avoid the vacuum suction out and bring the operation stability. 


\subsection{Anti-jamming design}

In the mechatronics integrated assembly device, it contains not only the strong electrical equipment, but also the weak current equipment. There will be interference between these devices, and the interference can be divided into signal interference and electromagnetic noise interference. If the internal interference of the system affects the normal operation of other devices, the components in the whole integrated equipment will produce substantial results, and the data and processing results may be biased, which will affect the reliability of the whole system. Therefore, anti interference must be added to the design process, and the independence between components is realized by isolation or interference elimination. For example, the purpose of eliminating interference can be achieved by cutting off the transmission path of radiation electromagnetic noise or shielding cable signals. At the same time, the way of grounding can be used to eliminate voltage difference by using reference location, so as to protect the whole electric control cabinet and improve its anti-interference ability.

The anti interference technology in the current, there is a more advanced method for filtering technology, composed of two port network through the resistor capacitor or ferrite devices, when inserted into the transmission line when the frequency will reduce the small weak, large attenuation frequency will be blocking. The anti-interference technology is not widely applied at present, but the market prospect is better. It is believed that in the near future, it will be improved step by step, and will be widely applied in the electrical control system of Mechatronics integrated assembly device.

\subsection{Heat design}

In a mechatronic integrated equipment device, many electronic components have the conditions for their use, such as temperature. If the electronic components exceed the limits of their temperature when they are used, the materials will undergo varying degrees of change, and their physical properties will be changed. Then the components will not play their intended role. Of course, there are certain rules for such failures in components. For high temperature and high negative conditions, the malfunctions of semiconductor components are directly proportional to the temperature. Under such conditions, their voltage and electrical performance parameters have changed. In view of this situation, PLC system can be used for control. By collecting the heat in the module to analyze whether or not it reaches the limit of components, if we reach the limit, we should take cooling measures, such as fans, to achieve cooling. When the temperature drop can not meet the actual demand, it can be cut off in order to stop the device and avoid damage caused by long time operation.

In the electrical system of a lot of Mechatronics integrated assembly devices, water cooling is used to design the cooling system at present. This design realizes the purpose of cooling by circulating liquid, which takes away the heat in the whole control system and avoids the damage of components caused by high temperature. In this design, there is no need to increase the monitoring system, because the heat will be lower than the environmental requirements of components, but the cost of such design is generally high, and additional circulating pumps need to be added, which are commonly used in the more precise Mechatronics integrated assembly devices.

Of course, for the mechatronics integrated assembly device, there are many other aspects of its optimization design. These need to be analyzed in combination with specific situations. The above three kinds are the main direction of improvement. In the future development, the level of science and technology will gradually improve, which can provide a more solid foundation for the corresponding design, and at the same time, it can provide more optimization directions. In the current social development, automation technology is constantly improving. The use of signal transmission and the judgement of the central processor can achieve independent control, and even self repair for some small faults. These are in the future in the electromechanical integrated assembly device of electric control the direction of development, I believe that with the continuous improvement of the control system, mechatronics integrated assembly device will be more perfect, to lay a solid foundation for controlling the quality of products and improve production safety. 


\section{The main advantages of the improved device}

After the optimization of the electrical system of the electromechanical integrated assembly assembly, the characteristics of the electrical system are mainly shown in the following aspects:

(1) the structure is more compact and simple. The structure of the improved control system has been greatly optimized, and the space occupied by it is smaller. All movements can also reduce the possibility of interference, which plays a great role in the improvement of hardware. Especially for more complex systems, it can simplify motion, save electrical energy, and greatly improve the efficiency of production.

(2) the difficulty of software development has been reduced in varying degrees. In the process of optimization design, its software eliminates the process of signal transmission and data processing, so the workload of developing software is not large, and the difficulty is low. It can well fit the current technology situation in China. Moreover, many improvements are done on the basis of the original software, simplifying the CNC system by removing complex programs, or simplifying the CNC system by merging. For the entire electrical control, this can maximize the cost of optimization.

(3) the reliability and safety of production have a great guarantee. After the improvement of the electric control system of the original, can eliminate the interference between the components, the operation is more stable, so the movement and control of more reliable and safe, low probability of the fault occurred in the production process, which has great significance to ensure stable operation of production.

(4) the service life is longer. In the optimized design, the effect of temperature on the life of the component is taken into account, so the control of cooling is added. After the precise control of the temperature, the use of various components is guaranteed, which reduces the failure to attempt in the process. At the same time, due to the compliance of the environment, the components of these devices can also avoid the unstable operation, and extend the life of all kinds of devices. The control is more reliable and the cost of maintenance is reduced to a certain extent.

\section{Conclusion}

With the rapid development of science and technology and social progress, China's current mechanical assembly industry is advancing rapidly, especially in science and technology continue to extend to the electrical control of Mechatronics integrated assembly device provides more powerful protection, a lot of products than the traditional way a great reform, in which the role played by more and human more small, the production efficiency has been greatly improved. However, in view of the actual situation, there is still room for optimization of the electrical control of the electromechanical integration integrated assembly device in our country. It can be improved from the aspects of safety, reliability, software control and line layout. To solve this problem, this paper makes a detailed analysis from the three aspects of redundancy design, anti-interference design and thermal design, and hope that through these studies to China's mechanical and electrical integration equipment electrical control to provide the corresponding direction of system optimization, promote sustainable development of the industry.

\section{References}

[1] Zheng Yuanming. Optimization and improvement design of electrical control system of electromechanical integrated assembly assembly [D]. University of electronic technology, 2007.

[2] Song Huawei. Analysis of the optimization of electrical control system of integrated mechanical and electrical integrated assembly device [J]. Intellect, 2011, (25): 75.

[3] Wang Chengxun. Optimization and improvement design of electrical system of Mechatronics integrated assembly device [J]. New technology and new products in China, 2011, (24): 163-164.

[4] Jiang Rongdong. Optimization and improvement of electrical system of Mechatronics integrated 
assembly device [J]. Industrial design, 2012, (02): 259.

[5] Zheng Zhaoquan. Mechatronics integrated assembly device electrical system optimization of [J]. science and Technology Economic Herald, 2017, (17): 93.

[6] Ren Hanxia . Analysis of electromechanical integrated assembly device of the electric control system optimization of [J]. city construction theory research (Electronic Edition), 2017, (21): 16-17. 